\title{
ANDES

www.scielo.cl

\section{Diabetes mellitus tipo 1 y enfermedad de Graves Basedow, un caso de Síndrome Poliglandular Autoinmune}

\author{
Type 1 diabetes mellitus and Graves Basedow's disease, a case of Autoimmune \\ Polyglandular Syndrome
}

Cindy Arteta-Acosta ${ }^{\mathrm{a}, \mathrm{b}}$, Jonathan Kraus ${ }^{\mathrm{b}}$, Natalia Galilea ${ }^{\mathrm{b}}$, Priscilla Prado ${ }^{\mathrm{b}}$, Marta Azocar $^{\mathrm{b}}$

anniversidad de Chile. Santiago, Chile

bServicio de Pediatría, Hospital Luis Calvo Mackenna. Santiago, Chile

Recibido: 4 de noviembre de 2020; Aceptado: 17 de marzo de 2021

\section{¿Qué se sabe del tema que trata este estudio?}

El Síndrome Poliglandular Autoinmune (SPA) es un grupo de desórdenes heterogéneos que combina varias enfermedades autoinmunes, se clasifican en cuatro tipos, siendo el tipo 1 el más frecuente en la infancia, el 2 en adolescencia y adultos, y los 3 y 4 más frecuentes en adultos.

\begin{abstract}
¿Qué aporta este estudio a lo ya conocido?
Presentamos el caso de un adolescente con SPA tipo 3A dado por Diabetes Mellitus I e hipertiroidismo con Enfermedad de Graves Basedow. En Chile la DM1 ha incrementado en los últimos años, por ello es importante buscar otras enfermedades autoinmunes en este grupo de pacientes.
\end{abstract}

\section{Resumen}

La diabetes mellitus 1 (DM1) es una de las patologías autoinmunes más frecuentes en la infancia. Su diagnóstico exige la búsqueda de otras enfermedades autoinmunes. Objetivo: Presentar el caso de un paciente pediátrico con dos enfermedades endocrinas autoinmunes de concomitancia poco frecuente. Caso Clínico: Adolescente masculino de 12 años, sin antecedentes mórbidos de importancia, es hospitalizado por cuadro clínico de 3 meses de evolución consistente en fatiga, dolor ocular, edema palpebral intermitente, bocio, polifagia, polidipsia, poliuria y pérdida de peso $(12 \mathrm{~kg})$, síntomas compatibles con DM1 asociado a enfermedad de Graves Basedow, lo cual se confirmó por medio de exámenes de laboratorio, glicemia elevada (207 mg/dL, HbA1C 10,9\%), TSH suprimida (<0,01 uUI/ $\mathrm{mL})$, T4L elevada $(6,99 \mathrm{ng} / \mathrm{dL})$ y presencia de autoanticuerpos anti-tiroperoxidasa, antitiroglobulina y anti-receptor TSH, con hallazgos ecográficos concordantes. Por lo anterior se realizó diagnóstico de Síndrome poliglandular autoinmune (SPA) 3A, se indicó tratamiento con insulina, propranolol y tiamazol. El paciente evolucionó de forma satisfactoria con control de síntomas y glicemias, egresando con control ambulatorio. Conclusión: Presentamos el caso de un adolescente que debutó con SPA por DM1 e hipertiroidismo en forma simultánea. Estos SPA pueden ser más comunes de lo que se reporta en la práctica clínica. La alteración de dos o más glándulas endocrinas u otras enfermedades autoinmunes debe hacernos sospechar su diagnóstico, lo cual tiene importante implicación clínica, como co-morbilidad y pronóstico de calidad de vida.
Palabras clave:

Diabetes Mellitus 1; Hipertiroidismo; Síndrome Poliglandular Autoinmune; Adolescente 


\section{Abstract}

Type 1 diabetes mellitus (T1DM) is one of the most frequent autoimmune diseases in childhood. Its diagnosis requires the search for other autoimmune diseases. Objective: to present the case of a pediatric patient with two rare concomitant autoimmune endocrine diseases. Clinical Case: A 12 -year-old male with no significant morbid history, is hospitalized due to a 3-month clinical picture of fatigue, eye pain, intermittent eyelid edema, goiter, polyphagia, polydipsia, polyuria, and weight loss (12 kilograms), compatible with T1DM and Graves-Basedow disease. It was confirmed by laboratory tests which showed elevated glycemia (207 mg/dL, HbA1C 10.9\%), suppressed TSH $(<0.01 \mathrm{uIU} / \mathrm{mL})$, elevated FT4 $(6.99 \mathrm{ng} / \mathrm{dL})$, and the presence of anti-autoantibodies thyroid peroxidase, antithyroglobulin, and anti-TSH receptor, along with suggestive ultrasound findings. Therefore, we established the diagnosis of autoimmune polyglandular syndrome (APS) 3A and initiated treatment with insulin, propranolol, and thiamazole. The patient evolved satisfactorily and was discharged with outpatient follow-up. Conclusion: We present the case of an adolescent who presented APS due to T1DM and hyperthyroidism. This APS may be more common than is reported in clinical practice. The alteration of two or more endocrine glands or other autoimmune diseases should make us suspect its diagnosis, with important clinical implications, such as comorbidity and quality of life prognosis.

\section{Keywords:}

Type 1 Diabetes

Mellitus;

Hyperthyroidism;

Autoimmune

Polyglandular

Syndrome; Adolescent

\section{Introducción}

La diabetes mellitus 1 (DM1), es una de las enfermedades crónicas más comunes en la infancia. El mayor peak de presentación ocurre entre los 5-7 años de edad y cerca de la pubertad, presentándose más temprano en las mujeres (entre 5-9 años) comparado con los hombres (10-14 años $)^{1}$. Su incidencia ha ido en aumento en los últimos años, y varía de acuerdo con la región. En China se ha descrito una incidencia de 0,1/100.000 habitantes, mientras que en Finlandia se describen más de 60 casos por 100.000 personas/año 2 . En Chile, la incidencia de DM1 pasó de 10,1/100.000 en menores de 20 años en el 2006 a 16,5/100.000 en menores de 20 años en el $2014^{3}$.

La DM1 frecuentemente se asocia con otras enfermedades autoinmunes endocrinas y no endocrinas, incluyendo hiper o hipotiroidismo, enfermedad celiaca, falla adrenal, enfermedad inflamatoria intestinal, enfermedad vascular del colágeno, entre otras ${ }^{4}$.

Las enfermedades tiroideas constituyen un problema de salud pública que puede sustancialmente impactar en el bienestar del paciente, particularmente en el embarazo y la infancia ${ }^{5}$. De estas, la más frecuente es el hipotiroidismo, con una prevalencia en la población general entre $0,2-5 \%{ }^{5}$. Las principales causas de hipotiroidismo primario son la deficiencia de yodo y enfermedades autoinmunes (tiroiditis de Hashimoto). Por su parte, la prevalencia global del hipertiroidismo se encuentra entre $0,2-1,3 \%{ }^{5}$, siendo la causa principal la enfermedad de Graves Basedow (EGB) en 50-80\% $\%^{5}$. De forma frecuente, ciertos síndromes se han relacionado con enfermedades tiroideas, como el síndrome de Down y síndrome de Turner5. Así mismo, un paciente con una enfermedad tiroidea por desorden autoinmune tiene más riesgo de presentar otras enfermedades autoinmunes como diabetes, alopecia, vitíligo y enfermedad celiaca ${ }^{6}$.

La coexistencia de más de una enfermedad autoinmune en un paciente se ha denominado síndrome poliglandular autoinmune (SPA). Los SPA son un grupo de desórdenes heterogéneos, que combinan varias enfermedades autoinmunes. Se caracterizan por la infiltración linfocítica que puede afectar tanto a órganos endocrinos como no endocrinos, la presencia de autoanticuerpos contra los órganos y un defecto en la respuesta inmune celular y humoral ${ }^{7}$. Los SPA han sido clasificados en cuatro tipos, siendo el tipo 1 el más frecuente en la infancia, el tipo 2 en adolescencia y adultos, y los tipos 3 y 4 más frecuentes en adultos 8 .

La prevalencia de los desórdenes autoinmunes ha ido en aumento en las últimas décadas ${ }^{7}$. En muchos casos, suelen ser considerados como dos entidades aisladas, en vez de comorbilidades, por ello se considera que hay un gran sub-registro de las mismas; por tanto, su verdadera prevalencia aún se desconoce ${ }^{7}$.

El objetivo de esta comunicación es presentar el caso de un paciente pediátrico con dos enfermedades endocrinas autoinmunes de concomitancia poco frecuente.

\section{Caso Clínico}

Adolescente de 12 años de edad, sin antecedentes mórbidos de importancia, consultó a un centro de atención primaria en salud por cuadro clínico de 3 meses de evolución consistente en sensación de fatiga, do- 
lor ocular y edema de párpados intermitente, asociado a polifagia, polidipsia, poliuria, nicturia y pérdida de peso aproximada de $12 \mathrm{~kg}$, sin síntomas gastrointestinales, respiratorios o fiebre. Se solicitaron exámenes con control posterior, destacando hiperglicemia, aumento de hemoglobina glicosilada, y alteración de las pruebas tiroideas: hormona estimulante de tiroides (TSH) disminuida, tetrayodotironina libre (T4L) elevada; glucosuria de $200 \mathrm{mg} / \mathrm{dL}$, perfil hepático, renal y electrolitos y ácido-base normal (tabla 1). Con estos resultados, se derivó al servicio de urgencias del Hospital Luis Calvo Mackenna.

Al examen físico, se encontró taquicárdico (140 latidos/minuto), hipertenso $130 / 79 \mathrm{mmHg}$ (percentil 95), peso $37 \mathrm{~kg}$, talla 1,62 metros, índice de masa corporal (IMC) 14,14, IMC/E -3,13 (Z), T/E -0,15 (Z). Así mismo, se observó exoftalmos (figura 1a) tiroides aumentada de tamaño bilateral de aproximadamente $3 \mathrm{~cm}$ de diámetro (figura $1 \mathrm{~b}$ y $1 \mathrm{c}$ ), no dolorosa, lisa, sin nódulos, no se auscultó soplo en tiroides, pero sí danza carotidea. Se objetivó temblor fino en manos a la extensión de los brazos y piel caliente. Los exámenes de ingreso confirmaron hiperglicemia, HbA1C elevada, sin cetonemia pero con cetonuria; hemograma, función renal, gases venosos y electrolitos sin alteraciones, TSH suprimida, triyodotironina (T3) y T4L elevadas (tabla 1).

En estudios de extensión se encontró anticuerpos anti-tiroperoxidasa (aTPO) elevados, anticuerpos antitiroglobulina (aTG) ligeramente elevados y anticuerpo estimulante del receptor TSH (TRab) positivos en $35.4 \mathrm{UI} / \mathrm{l}$ (tabla 1), con anticuerpos negativos para enfermedad celiaca (antiendomisio, antitransglutaminasa). No hubo alteración en electrocardiograma. En ecografía de cuello, se observó una tiroides de bordes lobulados y tamaño aumentado, lóbulo derecho $23 \mathrm{x}$ 22 x $62 \mathrm{~mm}$ y lóbulo izquierdo $22 \times 20$ x $60 \mathrm{~mm}$ en diámetros transverso, anteroposterior y longitudinal, respectivamente. Parénquima levemente hipoecogénico, de ecoestructura heterogénea y aspecto pseudonodular, con aumento en vascularización, sin lesiones focales sólidas ni quísticas; compatible con signos de tiroiditis crónica (figura 2a y 2b).

Por los hallazgos al examen físico y de laboratorio, el paciente se diagnosticó como debut de DM1 y enfermedad de Graves Basedow, recibió manejo con régimen diabético e insulina. Asimismo, para el tratamiento del hipertiroidismo se indicó propranolol y tiamazol.

Durante la hospitalización, el paciente evolucionó de forma favorable, con disminución de la presión arterial, frecuencia cardíaca y temblor en extremidades, requiriendo ajustes de su esquema de insulinas, logrando con ello alcanzar metas terapéuticas. Por tanto, se realizó diagnóstico de síndrome poliglandular auto-
Tabla 1. Exámenes de laboratorio

\begin{tabular}{lcc}
\hline Exámenes & $\begin{array}{c}\text { Centro atención } \\
\text { primaria }\end{array}$ & HLCM \\
\hline Glicemia $(\mathrm{mg} / \mathrm{dL})(\mathrm{VN}$ al azar < 140) & 155 & 207 \\
HbA1C $(\%)(5,7)$ & 12 & 10,9 \\
TSH $(\mathrm{uUl} / \mathrm{mL})(\mathrm{VN} 0,36-6,00)$ & $<0,085$ & 0,01 \\
T4L $(\mathrm{ng} / \mathrm{dL})(\mathrm{VN} 0,78-2,49)$ & 6,82 & 6,99 \\
T3 $(\mathrm{ng} / \mathrm{dL})(\mathrm{VN}(86-199)$ & - & 436 \\
aTPO $(\mathrm{Ul} / \mathrm{mL})(\mathrm{VN}<5,61)$ & - & 64,16 \\
aTG $(\mathrm{UI} / \mathrm{mL})(\mathrm{VN}<4,11)$ & - & 4,58 \\
TRab $(\mathrm{Ul} / \mathrm{l})(\mathrm{VN}<1)$ & - & 35,4
\end{tabular}

HbA1C hemoglobina glicosilada; TSH hormona estimulante de tiroides; T4L tetrayodotironina libre; T3 triyodotironina; aTPO anticuerpo tiroperoxidasa; aTG anticuerpo tiroglobulina; TRab anticuerpo contra receptor $\mathrm{TSH}$.

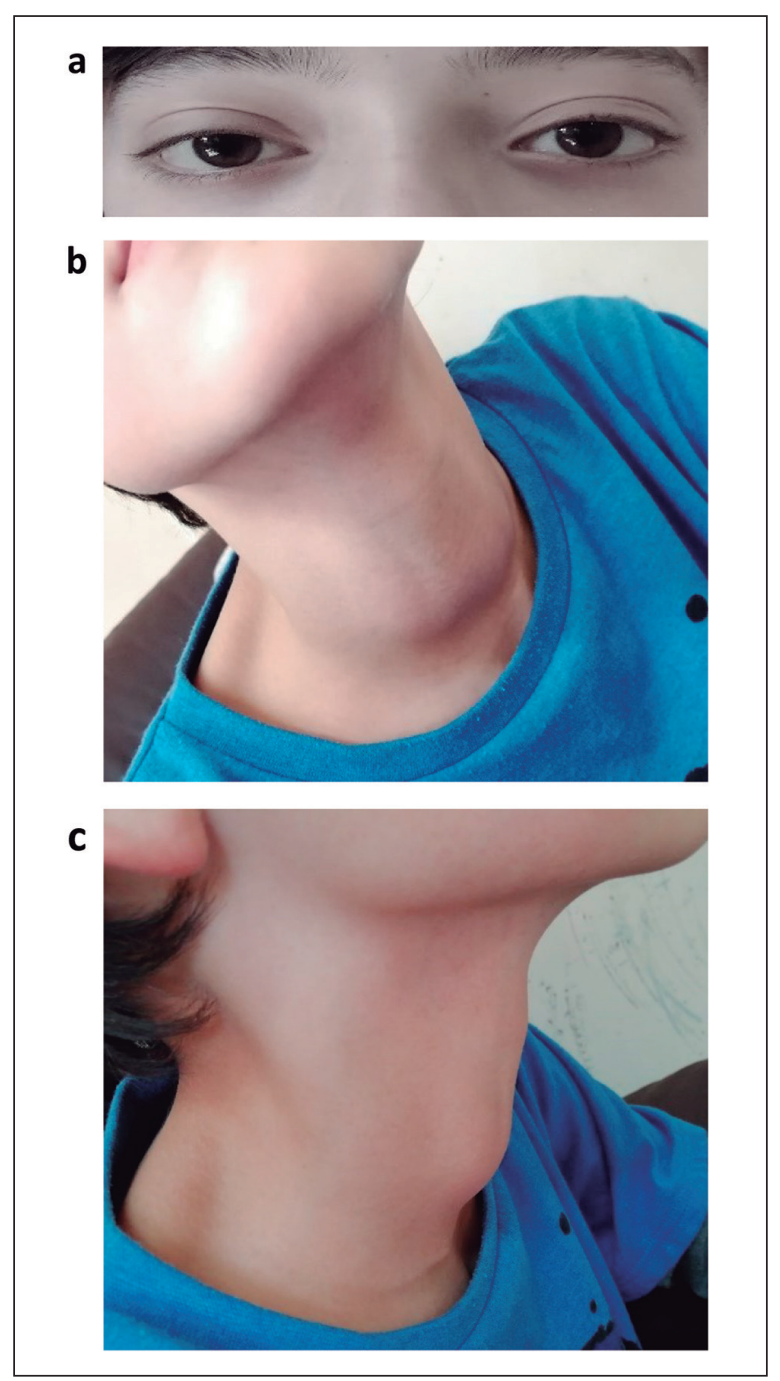

Figura 1. a: Foto frontal evidencia exoftalmo y tiroides aumentada de tamaño; b y c, fotos oblicuas y laterales que evidencian el marcado aumento de tamaño de la tiroides. 

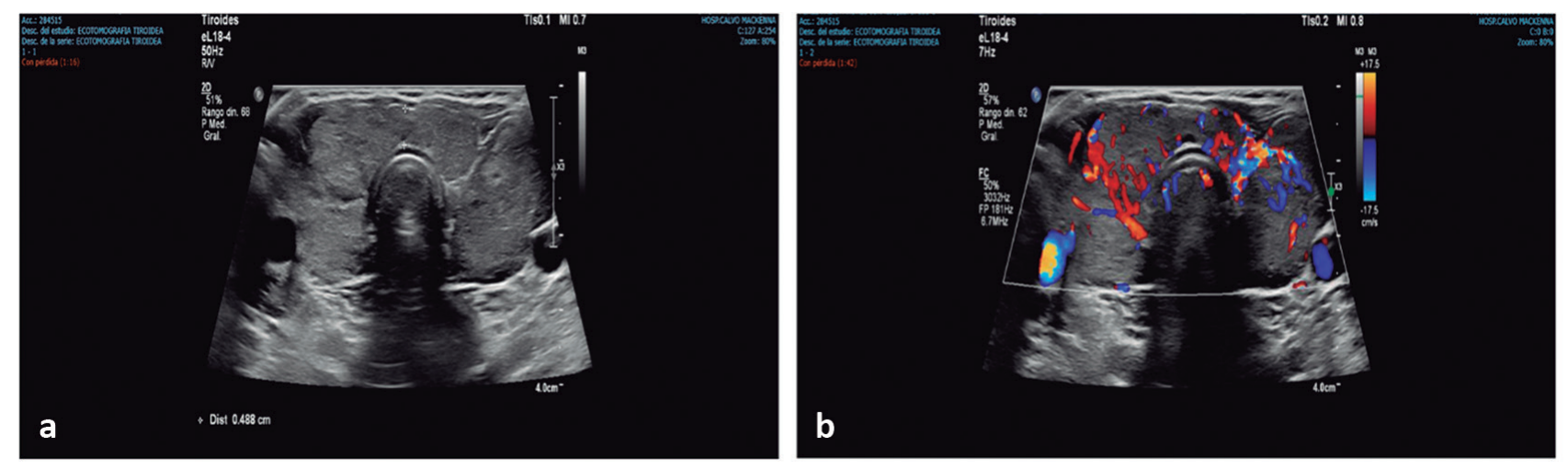

Figura 2. a: Ultrasonido de la glándula tiroidea, transductor lineal en escala de grises: glándula tiroidea aumentada de tamaño, bordes lobulados; b: transductor lineal con aplicación doppler color: aumento de la vascularidad en toda la glándula.

inmuno tipo 3A. Fue dado de alta luego de educación de sus enfermedades de base tanto a paciente como a familiar, en buenas condiciones clínicas, y se indicó seguimiento ambulatorio con equipo de endocrinología.

La publicación de este caso fue aprobada por el Comité de Ética de la Universidad de Chile.

\section{Discusión}

Se presenta el caso de un paciente adolescente con SPA tipo 3A establecido por la presencia de hipertiroidismo y DM1. El SPA es un desorden complejo y heterogéneo, muchas veces sub-diagnosticado debido a su amplia variedad de presentación ${ }^{1}$. La condición de base es una pérdida de la tolerancia inmune que conlleva al deterioro de la función poliglandular ${ }^{1}$.

El SPA fue descrito por primera vez en 1929 por Thorpe y $\mathrm{col}^{9}$ quienes establecieron la asociación entre hipoparatiroidismo y candidiasis mucocutánea, y a medida que se fueron describiendo mayor número de asociación de otras glándulas afectadas se fue aumentando el espectro de este síndrome ${ }^{8}$. De acuerdo a las glándulas endocrinas comprometidas, se ha clasificado en 4 tipos $^{8,10}$.

El SPA tipo 1 se caracteriza por la presencia de candidiasis mucocutánea crónica, hipoparatiroidismo e insuficiencia suprarrenal. Se ha descrito una secuencia cronológica para este síndrome, donde antes de los 5 años de edad se presenta la candidiasis, entre los 5 y 10 años el hipoparatiroidismo y antes de los 15 años la enfermedad de Addison ${ }^{8,10}$. La causa de este síndrome se considera que es monogénica, por mutaciones presentes en el gen AIRE (gen regulador de la inmunotolerancia a nivel del timo $)^{8,10}$. Usualmente se manifiesta en la edad pre-escolar 3-5 años o en la adolescencia temprana, y aunque se considera de los SPA más ra- ros (1:100.000), una alta prevalencia ha sido descrita en ciertos grupos poblacionales como Finlandia (1:25.000) y Cerdeña $(1: 14.000)^{11,12}$.

El SPA tipo 2 tiene una frecuencia de 1:20.000 personas, sobretodo en mujeres (razón hombre: mujer 1:3), siendo la mayor incidencia entre la tercera y cuarta década ${ }^{13}$. Se caracteriza por la deficiencia de dos o más glándulas, principalmente las suprarrenales, tiroides (síndrome de Schmidt) y/o DM1 ${ }^{14}$. Este síndrome está relacionado con alteración en los genes codificantes de proteínas reguladoras claves en el sistema inmune innato y adaptativo, principalmente con el antígeno leucocitario humano (HLA) como el alelo DR3 y DR4, así como también genes que codifican CTLA-4 ${ }^{8,11}$.

El SPA tipo 3 es el más frecuente, puede presentarse incluso en la edad pediátrica, especialmente durante la adolescencia, como el caso descrito. Tiene un espectro más amplio de presentación y está relacionado con compromiso del HLA, CTLA-4, y los genes de la proteína tirosin-fosfatasa no receptor tipo 22 (PTPN22), en diferentes grupos étnicos, especialmente asiáticos ${ }^{15}$. El principal órgano afectado por disfunción autoinmune es la tiroides, asociado con otras enfermedades autoinmunes, excluyendo la enfermedad de Addison $^{8,16}$. Debido a su amplio espectro se ha subdividido en ${ }^{16}$ : A. Asociado a otras enfermedades endocrinas (DM1, síndrome de Hirata); B. Asociado a enfermedades gastrointestinales autoinmunes (gastritis atrófica, anemia perniciosa, enfermedad celiaca, enfermedad inflamatoria intestinal, hepatitis autoinmune, cirrosis biliar primaria, colangitis esclerosante); C. Asociada a enfermedades piel/neuromuscular/hematopoyético (vitíligo, alopecia, trombocitopenia autoinmune, anemia hemolítica autoinmune, síndrome antifosfolípidos, miastenia gravis, esclerosis múltiple); D. asociado a enfermedades del colágeno/vasculitis (lupus, artritis 
reumatoide, artritis reactiva, esclerodermia, síndrome de Sjögren, vasculitis).

El SPA tipo 4 es un síndrome raro, que incluye todas las combinaciones clínicas que no se clasifican en SPA anteriores ${ }^{16}$.

La real prevalencia del SPA tipo 3 es desconocida. Un estudio ${ }^{4}$ realizado en 461 niños polacos con DM1 encontró una prevalencia de 14,5\% y del SPA tipo 3A un $11,1 \%$. Del total de niños diabéticos, el 10,4\% presentó Hashitoxicosis y 0,7\% enfermedad de Graves, siendo más frecuente en niñas que en niños, con una relación 3:14. En Estados Unidos, el hipertiroidismo fue identificado en el $0,5 \%$ de niños con $\mathrm{DM} 1^{17}$. Según el grupo etario, el SPA tipo 3A, se ha identificado en $19 \%$ en menores de 5 años, 5,1\% entre $6-10$ años, $6,2 \%$ entre 11-15 años y 22,7\% entre 16-18 años.

En el caso enseñado por la presencia de DM1 y EGB, se hizo el diagnóstico de un paciente adolescente con SPA tipo 3A. Debido al diagnóstico simultáneo de ambas entidades no podemos afirmar cuál se presentó primero, sin embargo, en pacientes con DM1 es frecuente la asociación de otras enfermedades autoinmunes como enfermedad tiroidea en $15-30 \%$; dentro de ellas se señala como la más frecuente al hipotiroidismo (25\%), mientras que el hipertiroidismo corresponde al $0,5-0,7 \%$ de los casos ${ }^{4}$. Otra enfermedad relacionada con la DM1 ha sido la enfermedad celiaca $(1,6-$ $16,4 \%)^{18}$. Una mayor prevalencia de esta asociación ha sido encontrada en la población japonesa donde más del 90\% de los pacientes con DM1 presentan enfermedad tiroidea autoinmune, y específicamente, en un grupo de 30 pacientes con DM1 y EGB, el 60\% de ellos desarrolló EGB antes del inicio de la DM1, y un 10\% presentó DM1 y EGB en forma concurrente ${ }^{15}$, lo cual podría ser el caso de nuestro paciente. Por otro lado, si se considera como base los pacientes pediátricos con enfermedad tiroidea autoinmune, se ha encontrado que un $30 \%$ de estos presentan compromiso de otra glándula endocrina, siendo la DM1 la más frecuente $(61 \%)$, seguido de la enfermedad celiaca $(22,2 \%)^{19}$. Por tanto, ante el diagnóstico de alguna de estas enfermedades autoinmunes es necesario hacer la búsqueda de las otras enfermedades, tal como fue explorado en nuestro paciente.

El diagnóstico de la DM1 se basa en los criterios clínicos y de laboratorio, no siendo necesaria la detección de los autoanticuerpos ${ }^{20}$. Igualmente, el diagnóstico de la EGB puede ser establecido rápidamente por medio de la clínica, con una historia de síntomas en los últimos meses en un paciente con cualquier signo de orbitopatía de Graves, bocio difuso y alteración de hormonas tiroideas (aumento de T4L, TSH suprimida). El predominio de la clínica hipertiroidea y la presencia de orbitopatía sugieren que este caso se trata de una enfermedad de Graves Basedow. La presencia de autoanticuerpos permite reforzar aún más el diagnóstico; por ejemplo, en la Hashitoxicosis son frecuentes los anticuerpos aTPO y aTG, mientras que en la EGB es más frecuente la presencia de TRab y aTPO ${ }^{21}$. En el caso presentado, se demostró la presencia de autoanticuerpos contra TRab, aTPO y aTG, los cuales de forma particular pueden ser positivos hasta en un 10\% de los pacientes con enfermedad de Graves ${ }^{21}$.

De acuerdo con lo anterior, el presente caso es excepcional, no solo por la concomitancia de DM1 e hipertiroidismo, sino también por ser un paciente de sexo masculino, adolescente y por la presencia de los anticuerpos positivos contra varios antígenos tiroideos. El manejo debe estar centrado en tratar las patologías de base de los pacientes, tal como es señalado en las guías nacionales y americanas ${ }^{20,22,23}$.

\section{Conclusión}

Presentamos el caso de un adolescente con SPA por DM1 y EGB en forma simultánea. Estos SPA pueden ser más comunes de lo que se reporta en la práctica clínica. La alteración de dos o más glándulas endocrinas u otras enfermedades autoinmunes debe hacernos sospechar su diagnóstico. Dada la frecuente asociación que se reporta entre DM1 y enfermedad tiroidea en pacientes pediátricos, es muy probable que se realice un sub-diagnóstico del SPA-3A, lo cual tiene importante implicación clínica, como comorbilidad y pronóstico de calidad de vida.

\section{Responsabilidades Éticas}

Protección de personas y animales: Los autores declaran que los procedimientos seguidos se conformaron a las normas éticas del comité de experimentación humana responsable y de acuerdo con la Asociación Médica Mundial y la Declaración de Helsinki.

Confidencialidad de los datos: Los autores declaran que han seguido los protocolos de su centro de trabajo sobre la publicación de datos de pacientes.

Derecho a la privacidad y consentimiento informado: Los autores han obtenido el consentimiento informado de los padres (tutores) del paciente y/o sujetos referidos en el artículo. Este documento obra en poder del autor de correspondencia

\section{Conflicto de intereses}

Los autores declaran no tener conflicto de intereses. 


\section{Referencias}

1. Hansen MP. Type 1 diabetes and polyglandular autoimmune syndrome: A review. World J Diabetes. 2015;6(1):67.

2. Atkinson MA, Eisenbarth GS, Michels AW. Type 1 diabetes. Lancet. 2014;383(9911):69-82.

3. Ochoa MF, García H. Terapia complementaria a la Insulina en el tratamiento de niños y adolescentes con Diabetes Mellitus tipo 1- (DM1). Rev Chil Endocrinol Diabetes. 2019;12(2):124-32.

4. Ben-Skowronek I, Michalczyk A, Piekarski R. Type III Polyglandular Autoimmune Syndromes in children with type 1 diabetes mellitus. Ann Agric Env Med. 2013;20(1):140-6.

5. Taylor PN, Albrecht D, Scholz A, et al. Global epidemiology of hyperthyroidism and hypothyroidism. Nat Rev Endocrinol. 2018;14(5):301-16

6. Hanley P, Lord K, Bauer AJ. Thyroid Disorders in Children and Adolescents: A Review. JAMA Pediatr. 2016;170(10):1008.

7. Pham-Dobor G, Hanák L, Hegyi P, et al. Prevalence of other autoimmune diseases in polyglandular autoimmune syndromes type II and III. J Endocrinol Invest. 2020;43(9):1-9.

8. Navarrete-Tapia U. Síndrome poliglandular autoinmune. Rev Med Hosp Gen Méx. 2013;76(3):143-52.
9. Thorpe E. Chronic tetany and chronic mycelial stomatitis in a child aged four and one-halfs years. Arch Pediatr Adolesc Med. 1929;38(2):328.

10. Letelier M. Síndromes endocrinos autoinmunes: cuándo sospechar y estudiar un síndrome poliglandular (SPG). Rev Med Clin Condes 2013;24(5):784-9.

11. Husebye ES, Anderson MS, Kämpe O. Autoimmune Polyendocrine Syndromes. Ingelfinger JR, editor. N Engl J Med. 2018;378(12):1132-41.

12. Husebye ES, Perheentupa J, Rautemaa $\mathrm{R}$, et al. Clinical manifestations and management of patients with autoimmune polyendocrine syndrome type I. J Intern Med. 2009;265(5):514-29.

13. Kahaly GJ. Polyglandular autoimmune syndromes. Eur J Endocrinol. 2009;161(1):11-20.

14. Betterle C, Dal Pra C, Mantero F, et al. Autoimmune Adrenal Insufficiency and Autoimmune Polyendocrine Syndromes: Autoantibodies, Autoantigens, and Their Applicability in Diagnosis and Disease Prediction. Endocr Rev. 2002;23(3):32764.

15. Horie I, Kawasaki E, Ando T, et al. Clinical and Genetic Characteristics of Autoimmune Polyglandular Syndrome Type 3 Variant in the Japanese Population. J Clin Endocrinol Metab. 2012;97(6):E1043-50.
16. Betterle C, Zanchetta R. Update on autoimmune polyendocrine syndromes (APS). Acta Bio Med. 2003;74(1):9-33.

17. Barker JM. Clinical review: Type 1 diabetes -associated autoimmunity: natural history, genetic associations, and screening. J Clin Endocrinol Metab. 2006;91:1210-7.

18. American Diabetes Association. 13. Children and Adolescents: Standards of Medical Care in Diabetes-2019. Diabetes Care. 2019;42(Supplement 1):S148-64.

19. Valenzise M, Aversa T, Saccomanno A, et al. Epidemiological and clinical peculiarities of polyglandular syndrome type 3 in pediatric age. Ital J Pediatr. 2017;43(1):69.

20. American Diabetes Association.

2. Classification and Diagnosis of Diabetes: Standards of Medical Care in Diabetes-2019. Diabetes Care. 2019;42(Supplement 1):S13-28.

21. Srinivasan S, Misra M. Hyperthyroidism in Children. Pediatr Rev. 2015;36(6):23948.

22. MINSAL. Guía clínica Diabetes Mellitus tipo 1.2013

23. Ross DS, Burch HB, Cooper DS, et al. 2016 American Thyroid Association Guidelines for Diagnosis and Management of Hyperthyroidism and Other Causes of Thyrotoxicosis. Thyroid. 2016;26(10):1343-421. 